Original Research Article

\title{
A prospective analysis of adherence to standard treatment guidelines in management of snakebite in a tertiary care teaching hospital of rural Bengal, India
}

\author{
Sonai Mandal ${ }^{1}$, Ananya Mandal ${ }^{1 *}$, Yendrila Chatterjee ${ }^{1}$, Somenath Das ${ }^{1}$, Syamal Kundu ${ }^{2}$, \\ Syed Mohammad Naser ${ }^{1}$
}

${ }^{1}$ Department of Pharmacology,

${ }^{2}$ Department of Medicine, Bankura Sammilani Medical College, Bankura, West Bengal, India

Received: 16 March 2019 Accepted: 25 March 2019

*Correspondence to:

Dr. Ananya Mandal,

Email:

drananyamandal@gmail.com

Copyright: (C) the author(s), publisher and licensee Medip Academy. This is an openaccess article distributed under the terms of the Creative Commons Attribution NonCommercial License, which permits unrestricted noncommercial use, distribution, and reproduction in any medium, provided the original work is properly cited.

\begin{abstract}
Background: Rural Bengal has one of the highest snakebite prevalence in India. This prospective observational study was initiated with the motive to analyse the effects and adherence to implementation of the new national snakebite protocol 2016.

Methods: Data were collected from the patients admitted with snake bite on a pre-designed and pre tested case study form and analysed with the help of descriptive statistics.

Results: A total of 75 patients arrived in the hospital during the study period out which 53 were male (most in the age group of 31-40 years) and majority of them were farmers $(26.6 \%)$ or housewives $(25 \%)$. Maximum of them were bitten in the lower limbs (54.6\%) and peak time of bite was between 6 am-12 pm (40\%). Most of them reached the hospital late, i.e., beyond 2 hours $(70.6 \%)$. It was found that there were 26 neurotoxic, 16 hemotoxic and 1 neuro-hemotoxic cases and the rest were non-venomous cases. Fifty-one of them received ASV and 4 died. There was $80 \%$ adherence in the indication of ASV administration, $73.3 \%$ in the ASV dose, $32 \%$ in the monitoring after ASV administration, $94.66 \%$ in the monitoring $20 \mathrm{WBCT}, 100 \%$ in adherence to haemodialysis as indicated.

Conclusions: The results showed good adherence in all the parameters. Protocol guided treatment has also resulted in rational use of ASV. So, from this study, it was concluded that protocol guided treatment is effective in snakebite management, but its continuous revision and amendments should be considered in future.
\end{abstract}

Keywords: Anti snake Venom (ASV), Hematotoxic envenomation, Snakebite, Standard treatment guidelines 2016, Neurotoxic envenomation

\section{INTRODUCTION}

Snakebite, being an acute life threatening, time-limiting medical emergency, poses a significant health concern especially in rural population of tropical and subtropical countries for which designing of a region-specific multidisciplinary protocol for snakebite management based on medical capacity of each country is always recommended. ${ }^{1,2}$ In 2009, snakebite was included in the list of neglected tropical disease by the World Health Organization (WHO). ${ }^{3}$ Fatality due to snakebite is due to wide species variation, shortage of anti-snake venom (ASV), poor compliance with treatment protocols, lack of public awareness and a clear policy to deal with the problem. 
Nearly 400000 people are bitten by snakes in a year and among those 82000 suffer from envenomation and 11,000 die. ${ }^{4}$ West Bengal is one of the highest snakebite prevalence states of India besides Andhra Pradesh, Kerala, Tamil Nadu and Maharashtra showing high average annual mortality rate in rural Bengal. ${ }^{5-7}$ But this data is widely believed to be an under-reported. ${ }^{8,9}$ India reports highest mortalities due to snakebite. ${ }^{10}$

In India, polyvalent ASV is produced by hyper immunising horses against venoms of four common species of snake i.e. the "Big Four" believed to be responsible for most deaths due to snakebite envenomation. ${ }^{10,11}$ However, the validity of the concept of "the big four" is increasingly challenged by the discovery that other important species in certain regions. ${ }^{10,11}$ Anti snake venom is recognized as an essential medicine and its timely administration with or without adjunctive treatment as needed prevents or reverses most of the manifestations of venomous snakebite. ${ }^{2,12}$

The main constraining factor till date remains the limited availability of ASV and its irrational use though poor access to health care services, difficult transportation and consequent delay in ASV administration remains to be the other key reasons behind high fatality rate in our country.

Irrational administration of ASV when not indicated results not only in wastage of resources but also exposing the patient to toxicity risk. Timely intervention and treatment can reduce both morbidity and mortality for which a need was felt for a National policy to be formulated with uniform implementation to ensure prompt availability and effective use of ASV in the rural areas of the country along with training and up gradation of knowledge of treating physicians to deal with emergencies. $^{2,13}$

Due to the prevailing practices in India where doses of ASV were uncertain, the Ministry of Health and Family Welfare (Government of India) in June 2016, formulated the National Protocol on Snake Bite Management besides the WHO Guidelines and standardized a treatment guideline for the effective management of snakebites in which training programs for care providers according to the new protocol were to be implemented..$^{3,7}$

Though the newly revised protocol introduced in 2016 proves to be cost effective but still there lies a gap in its proper implementation. Till date there are very few recent studies looking for its adherence and its relation to its outcome, but such study was never conducted in Bankura district of West Bengal which has a high prevalence of snakebites and related deaths..$^{8,13}$

The objective of this study was to evaluate the adherence to the guidelines of the new protocol on snakebite management prospectively so that the benefits and the drawbacks of the new protocol could be evaluated.

\section{METHODS}

The study was an Institution based prospective observational study conducted in the Department of Medicine and Pharmacology of Bankura Sammilani Medical College, Bankura, West Bengal - a tertiary care teaching hospital in rural Bengal, India. All patients with who presented themselves with any type of suspected snake bite symptom in the emergency and medicine unit of Bankura Sammilani Medical College and Hospital within the study period and were included in the study. The patients admitted in the hospital were divided into the three categories based on the clinical presentations.

- Nontoxic: Symptoms of anxiety, palpitations, tachycardia and paraesthesia,

- Neurotoxic: Symptoms of ptosis, diplopia, dysarthia, dysphonia, dyspnoea, dysphagia and paralysis,

- Hemotoxic: Symptoms of bleeding, demonstrable coagulopathy (20 WBCT in coagulable), shock and acute kidney injury (AKI).

Those patients who were brought dead or died upon arrival before receiving any treatments were excluded from the study. Those patients who were not willing to sign the informed consent document were also excluded.

The study was carried out during the period of 2 months, i.e., May and June of 2018 as snakebite is more prevalent in the monsoon and pre-monsoon in these areas. All data including demographic details, time of snake bite, time of hospital arrival and site of snakebite were entered in a predesigned and a pre-structured case study form. Patient relatives were also interviewed for this purpose.

The records of any outside treatment, setting and qualification of treating doctor were also taken into account. The details of first aid received including indoor treatment, door-to-needle time (defined as time from hospital arrival to administration of first treatment), requirement of antidote, including reasons for the same, its availability and their hospital stay were noted. The adherence parameters were grouped under the following categories.

- $\mathrm{ASV}$ indication and dose in accordance to different types of snakebite,

- $\quad$ Monitoring of the patient before and after receiving ASV,

- $\quad$ Atropine and neostigmine administration according to indication,

- $\quad$ Performing $20 \mathrm{WBCT}$ during diagnosis.

For neuroparalytic snakebite the dose of ASV was 10 vials stat as infusion over 30 minutes followed by second dose of 10 vials if no improvement was seen within first hour. Second dose can even be repeated after 2-3 hours if relapse of signs of neurotoxicity was noted though the maximum dose was 20 vials of ASV. ${ }^{6}$ 
In case of vasculotoxic snakebite, two regimens are followed-low dose infusion therapy and high dose intermittent bolus therapy. For high dose intermittent bolus therapy, 10 vials of polyvalent ASV given stat over 30 minutes as infusion, followed by 6 vials 6 hourly as bolus therapy till clotting time normalizes and/or local swelling subsides. Criteria for repeating dose would be detection of blood coagulability after 6 hours from first dose by 20 WBCT. 20 WBCT should be monitored every hour for first 3 hours and every 4 hours for remaining 24 hours. In case the test is non-clotting, it is to be repeated 6 hours after administration of loading dose of ASV. ${ }^{6}$

All patients who received ASV should be watched carefully every $5 \mathrm{~min}$ for first $30 \mathrm{~min}$, then at $15 \mathrm{~min}$ for 2 hours for manifestation of reaction. Atropine and neostigmine should be given to every patient with neurotoxic envenoming. Children and pregnant women must receive exactly the same dose of anti-venom as adults. Any deviation from the above-mentioned criteria were marked as non-adherence to the STG. ${ }^{6}$

After compiling the data using Microsoft Excel, the results were calculated and analysed with the help of descriptive statistics. The study was conducted after getting approval from Institute Ethics Committee and confidentiality of data was ensured.

\section{RESULTS}

A total of 75 patients were admitted with snake envenomation in the emergency and medicine department of Bankura Sammilani Medical College, West Bengal, India between $1^{\text {st }}$ May 2018 and $30^{\text {th }}$ June 2018. Among the 75 patients, $53(70.6 \%)$ patients were males. Most of the patients were middle aged of $31-40$ years (19 or $25.3 \%$ ), followed by 18 or $24 \%$ in the age group of $41-50$ years.

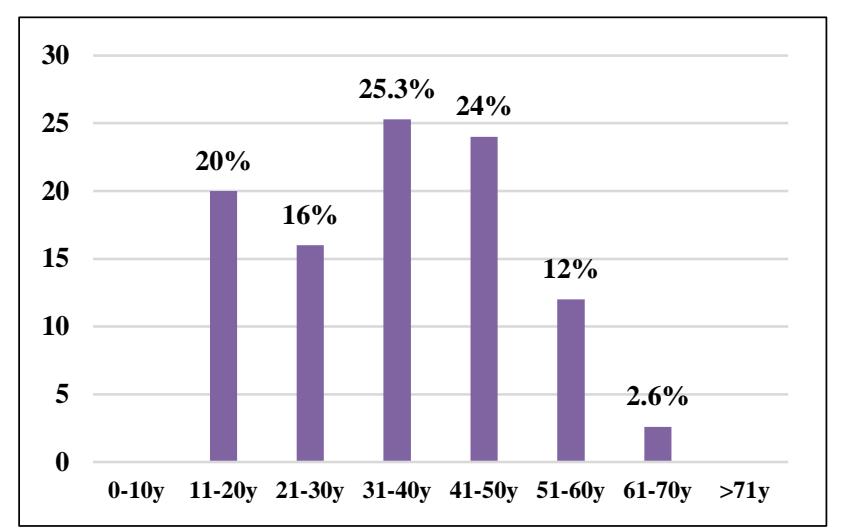

Figure 1: Age distribution of the patients.

Figure 1 Majority of the patients 59 (78.6\%) belonged to the rural area of Bankura and the rest from urban background. Major occupations among the patients were farming (20 or 26.6\%) and household work (19 or 25.3\%). Figure 2 The foot accounted for the commonest site of bite ( 23 or $30.6 \%$ ), followed by bites on leg $18.6 \%$ (14 cases), $\operatorname{arm} 12 \%$ (9 cases). Maximum number of bites took place in early morning from 6 am to 12 pm (30 cases or 40\%), followed by bites at night within 12 am to 6 am (22 cases or $29.3 \%$ ), evening $6 \mathrm{pm}$ to 12 am (15 cases or $20 \%)$ etc.

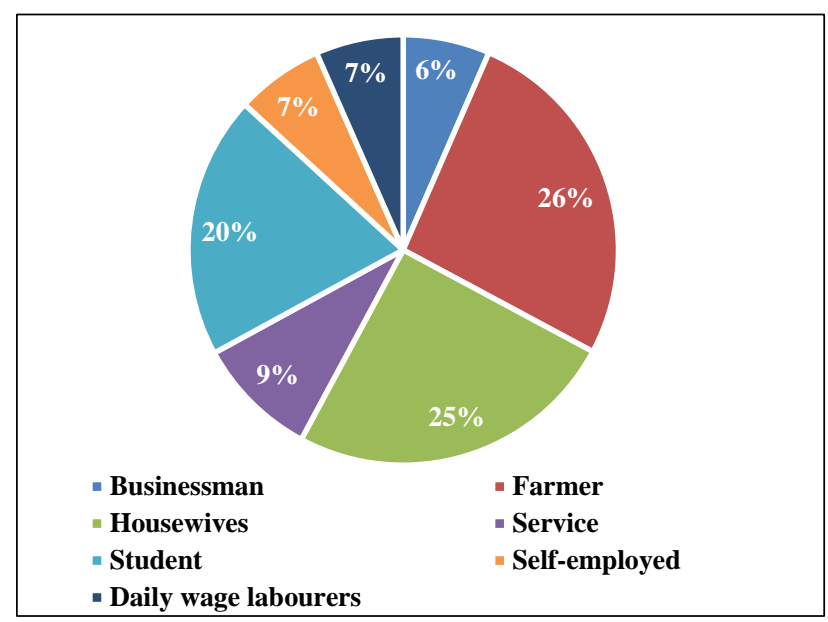

Figure 2: Distribution of the patients according to occupation.

A total of $32(42.6 \%)$ cases of nonvenomous snakebite and $43(57.3 \%)$ cases of venomous snakebite were registered. Among the venomous cases, 16 were hemotoxic $(27.9 \%)$, 26 neurotoxic $(45.3 \%)$ and 1 neuro-hemotoxic $(1.74 \%)$ cases.

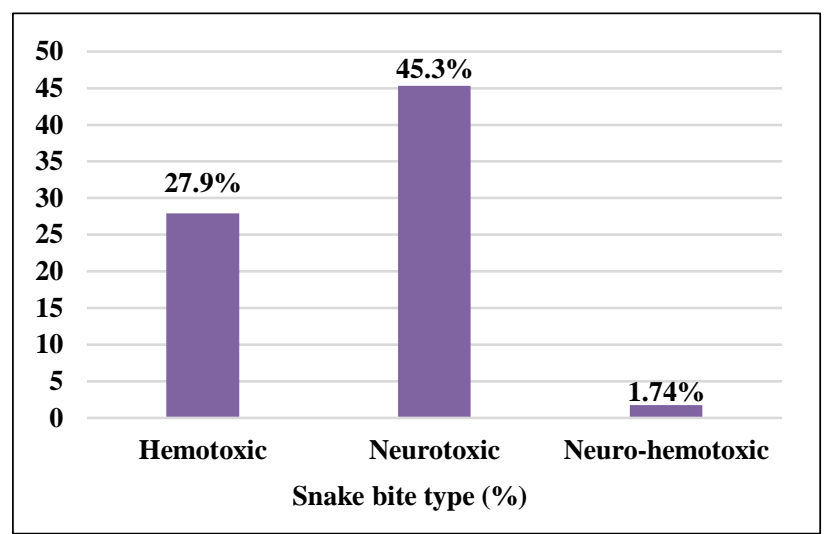

Figure 3: Envenomation profile of the patients.

Figure 3 among 75 patients, majority took more than 2 hours to arrive in the hospital (53 cases or 70.6\%) followed by $29.3 \%$ patients who arrived within 2 hours (22 cases) and $5 \%$ patients who arrived within 1 hour from the time of bite (4 cases) (Figure 4).

Symptoms and signs of presentation at the emergency department included $14(18.66 \%)$ cases of pain abdomen, $6(8 \%)$ cases of bleeding, $27(36 \%)$ cases of local swelling, $17(22.66 \%)$ cases of ptosis, $13(17.33 \%)$ cases of dysphagia, $17(22.66 \%)$ and cases of respiratory distress. No cases of DIC and compartment syndrome (Figure 5). 


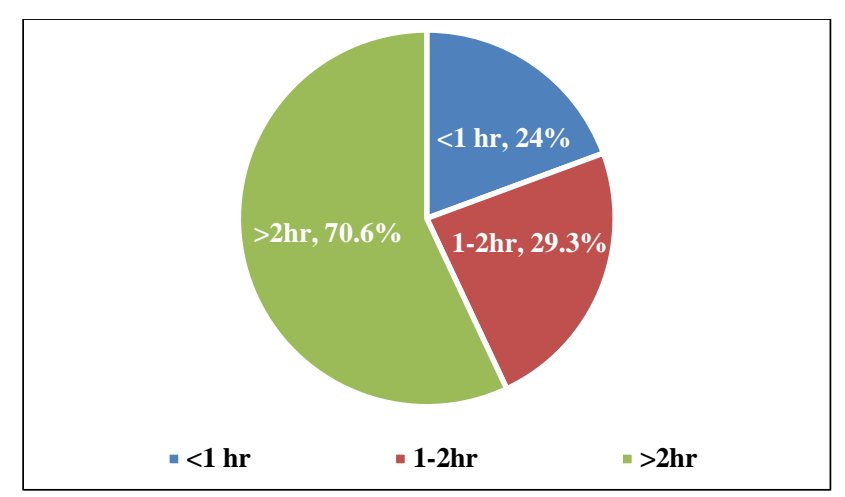

Figure 4: Distribution of patients according to the delay in presentation after bite.

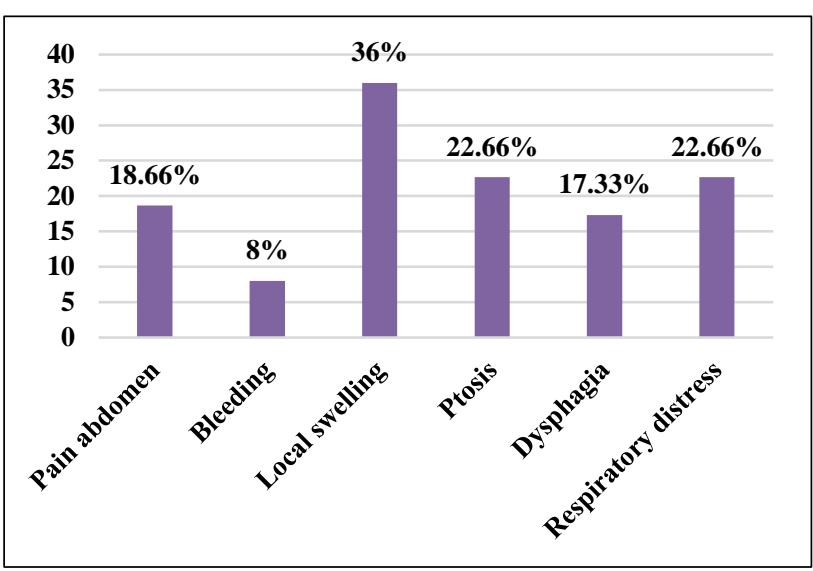

Figure 5: Distribution of patients according to the signs and symptoms on presentation after bite.

\section{Patient outcome profile}

Out of total 75 patients, $65(86.6 \%)$ were discharged from the hospital after full recovery. Among 65 patients 25 (33.33\%) were discharged within 2 days, 32 (42.6\%) patients were admitted for 3-4 days, $11(14.6 \%)$ patients were admitted for 5-7 days and $7(9.3 \%)$ patients were admitted for more than 7 days.

\section{ASV usage}

It was noted that 51 out of 75 patients admitted in emergency received ASV i.e. 68\%. Among the 32 non venomous cases, $9(28.1 \%)$ patients received ASV and among the 43 venomous cases, 42 (97.67\%) patients received ASV (Figure 6). 25 (96.1\%) patients out of 26 neurotoxic cases received ASV, all 16 patients (100\%) of hemotoxic bite received ASV and the 1 patient (100\%) of neuro-hemotoxic type also received ASV. A total of 1010 vials of ASV were utilised by all 51 patients of snakebite envenomation.

\section{ASV reactions}

All the patients were given Inj. hydrocortisone and Inj. adrenaline before administering ASV to avoid any incidence of severe anti-snake venom reaction. Only 1 $(1.33 \%)$ out of 75 patients suffered from anaphylactic reaction the rest were uneventful.

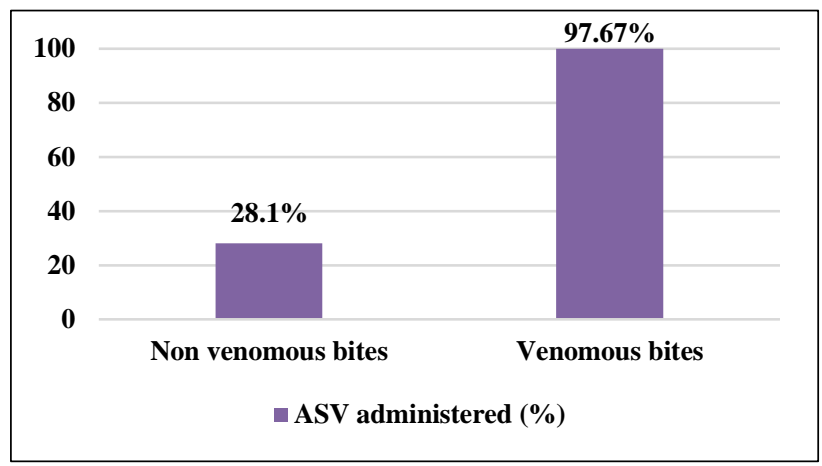

Figure 6: ASV administration among snake bite envenomation patients.

According the national snakebite protocol 2016, there was $80 \%$ adherence in the indication of ASV administration, while $73.3 \%$ adherence in the ASV dose but only $32 \%$ adherence in the monitoring after ASV administration. There was $94.66 \%$ adherence in the monitoring 20 WBCT. Adherence to atropine neostigmine administration was $80.76 \%$ in accordance to the mentioned indications (Figure 7).

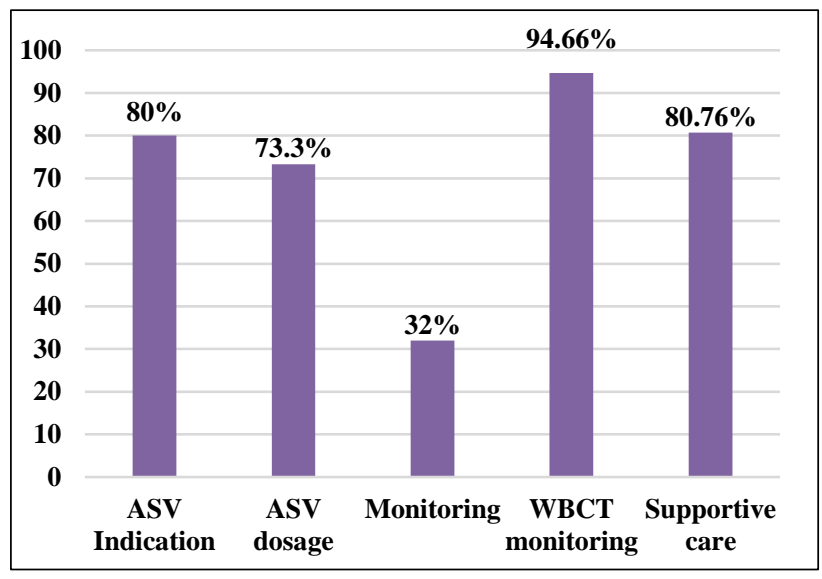

Figure 7: Adherence to protocol among the admitted patients.

\section{Complications and outcome}

It was seen that $7(9.3 \%)$ patients had to be put on dialysis and $9(12 \%)$ patients were put on ventilator. All the $4(6 \%)$ patients who died while under treatment in hospital were victims of venomous snakebite and 3 among them died due to respiratory failure (neurotoxic snakebites) and 1 due to AKI (hemotoxic snakebite). All 4 of them had a history of delayed reaching to the hospital by 2 hours or more. Moreover, all the 22 snakebite victims who reached the hospital before 2 hours had complete recovery with less duration of hospital stay and no complication. None among them had a hospital stay of more than 4 days thus also 
decreasing their cost of treatment. All the 18 patients who stayed for more than 5 days had history of late presentation in the hospital delaying their early intervention. Similar history was also found for the complicated cases such as patients on dialysis and ventilation $(12 \%)$.

\section{DISCUSSION}

In India, due to scarce supply of ASV in respect to demand, a more judicious use is needed. To have uniformity in treatment, a new revised National Snakebite Protocol was developed and adopted in 2016 which gave detailed guidelines regarding management. In this study, the adherence to the guidelines in the management and outcome in BSMC was studied.

A total of 75 snakebite patients who were admitted in the emergency and medicine department within the 2-month time frame of which 43 were diagnosed as venomous bite and remaining 32 as nonvenomous. Among 43 venomous snakebite victims 26 patients showed signs of neurotoxicity, 16 showed hemotoxic features and only 1 represented with features of both neurotoxicity and hemotoxicity. Hemotoxic bites were attributable to vipers, neurotoxic to cobra bites and simultaneous neurotoxic and hemotoxic manifestations in patients were attributable to kraits. $^{14-16}$

Most of the bites in this study population occurred in male population like the findings of other studies though the percentage of male vs female bites varied considerably. ${ }^{13,14}$ A wide variation of $70.6 \%$ vs $29.3 \%$ was found in male versus female were found in this study. This disparity is mainly because majority of rural males unlike female are more occupied in outdoor activities like farming where they are more exposed to snakes. The females were mainly bitten while sleeping at night or doing household chores.

The victims who were bitten at night gave a history of sleeping on floor bed and were bitten mostly by krait due to their active nocturnal habits unlike vipers and cobra which are generally active during day. Their bites are common while watering plantation or walking bare foot. ${ }^{17}$ A major portion of this study population is rural based (78.6\%) where there is lack a proper housing and a habit of sleeping on the floor which predisposes them to snakebite.

It has been found that the frequency of bite is more in the working age group of 31-50 followed by the 11-20 years age grouped school students who were mostly bitten while playing. Majority of bite were lower limb bites followed by arms. Bites on other areas during sleeping on floor and doing household activities corroborated with other studies. ${ }^{11,18} 70.6 \%$ of patients delayed in reaching hospital by more than 2 hours reflecting the problem of difficult transportation and lack of awareness in rural India. ${ }^{1,13,19}$ Among them 44\% were referred to BSMC from primary health care centres due to lack of ASV and required infrastructure.
In ASV outcome and delay in administration, ASV was administered correctly in $68 \%$ of snakebite victims. Among them 42 venomous snakebites received ASV except one patient who did not show any features of envenomation. He had a history of krait bite for which he was observed for appearance of signs and symptoms for 2 days. The reason for nonappearance of envenomation features may be that the bite might have been a dry bite, or he may have misidentified the snake. Among the 32 nonvenomous bites, 9 received ASV as the presenting features mimicked early envenomation. The striking similarity of signs of early envenomation and sympathetic overstimulation due to fear posed difficulties for the clinicians. $^{20}$ Severity and complications increased with increased 'Bite-to-ASV time' which is the time duration from the occurrence of bite to ASV administration. This also reflected in the outcome where all the 4 patients who died, were delayed in reaching the hospital. Among them 3 neurotoxic patients who died, the cause was the rapid onset of the symptoms and development of respiratory failure resulting from delay in reaching hospital. ${ }^{13}$ One patient died with hemotoxic features due to acute kidney failure proving snakebite treatment commencing earlier in medical facilities close to the victim can reduce the precious time loss in travel. ${ }^{13}$ An early and adequate institution of ASV as recommended in STG 2016, proved to be beneficial in early recovery as all the 22 patients who arrived early fully recovered without any complications and were discharged within 2 days. Patients, arriving late, with delayed administration of ASV required intensive therapies. ${ }^{21-23}$ Thus, there is an emergent need to spread awareness among the community for avoidance of any delay in treatment in snakebite.

The commonest clinical finding in the present study was local swelling (36\%) followed by ptosis, respiratory distress, dysphagia and pain abdomen which all points towards neurotoxic envenomation. Unlike ptosis, local swelling is found in both venomous and nonvenomous bites. Only $8 \%$ of patients presented with bleeding which is a sign of hemotoxic envenomation. This huge disparity of percentage of features of neurotoxic to hemotoxic is due to the speed of onset of symptoms. The signs and symptoms of neurotoxic bite are easily noticeable by both the patients and the health care givers for the rapidity of its onset. Failure of treatment by traditional healer becomes evident more quickly in neurotoxic bite than hemotoxic one. Similar finding regarding presenting features was also reported in a study done in Midnapore district of West Bengal. $^{13}$

With regards to adherence to STG in this study, it was found that the adherence to the indications for ASV administration according to STG was $80 \%$. The nonadherence was due to confusing and inapplicable grounds for determining if a patient was actually envenomed, late signs of appearance of envenomation and lack of knowledge about the local species of snakes. 
Adherence to ASV dose according to STG was $73.3 \%$. The non-adherence here was mainly due to cases of nonvenomous bites where ASV was given because of difficulty in discriminating features of early envenomation. Non adherence was also found in few patients who were referred from other primary health centers where the initial ASV dose given was not properly documented.

The adherence to ASV dose monitoring was 32\%. Low monitoring is due to a significant low doctor to patient ratio due to the fact that this hospital covers wide area of rural population. Though all the physicians were trained in STG 2016, but the all the nursing staff were not. With the ongoing regular training programs organized by the institution and government, soon all the health care givers will be trained in STG. Monitoring was mainly done in venomous cases and less frequently in non-venomous cases. There was a good adherence to STG in the proper hemodialysis and its indications and in the $20 \mathrm{WBCT}$.

Thus with regards to the result of implementation of the new STG, this study shows significantly good adherence in all the adherence parameters except the ASV monitoring which is achieved in less than 2 years of implication of the protocol and that too in a rural hospital. Protocol guided treatment has also led to a rationalised use of ASV. Earlier in BSMC, all the patients arriving in the hospital with the presentation of snakebite were given ASV without judging their toxicity based on a survey conducted in BSMC in 2016 where all snakebite patients were given ASV. ${ }^{24}$ But after the implementation of the protocol, no patients (except in those cases where indications were unclear) without proper indication of venomation was given ASV. As no study on ASV utilization was done in BSMC before 2016, comparison of ASV utilization was not possible. But a similar study conducted in Midnapore Medical college, where they introduced a local standardised protocol as an interventional tool, showed that protocol guided treatment resulted in a $66 \%$ decline in the amount of ASV administered to victims. ${ }^{13}$ This implementation can decrease the cost of treatment and also address the present problem of ASV crisis. ${ }^{21-23}$ More studies should be done to estimate the cost effectiveness of snakebite management. The mortality is $5.3 \%$ which also could have been reduced with early hospitalisation and ASV administration.

The significant limitation of this study was that the duration of this study period was only 2 months which did not include the monsoon when the bites peak. Moreover, author gathered data from only those patients who presented themselves in this hospital thus excluding those who were not referred to this hospital from PHCs and those who were treated by traditional healers.

\section{CONCLUSION}

Present study showed good adherence (more than $70 \%$ ) to the guidelines of the revised Standard treatment guidelines 2016 except with regards to monitoring of ASV for which a more intensified training program for all health care givers should be ensured. For a rural based hospital, achieving such adherence rate within 2 years of implementation of the protocol is a huge achievement which was possible only by continuous training and awareness.

Moreover, the protocol has also led to a rational use of ASV. Optimising ASV usage by developing a low dose treatment protocol which is equally beneficial and cost effective should be encouraged to overcome the ASV shortage.

The findings in this study will help the planners and policy makers in near future to know the areas to be given more importance to, i.e., proper training and workshops, development of more improvised local protocols considering the geographical changes and availability of required infrastructures. During this study, a need was felt for a proper guideline for PHCs which acts as the first treatment centre for snakebite victims. The government and the concerned authorities should take more initiation towards the production of more ASV to address its scarcity crisis.

\section{ACKNOWLEDGEMENTS}

Authors would like to thank faculty and staff of the Departments of Medicine and Pharmacology for assisting during study.

Funding: No funding sources

Conflict of interest: None declared

Ethical approval: The study was approved by the Institutional Ethics Committee

\section{REFERENCES}

1. Kulkarni ML, Anees S. Snake venom poisoning: experience with 633 cases. Ind Pediatrics. 1994;31:1239-43.

2. Warrell DA, Looareesuwan S, Stimson AF, Hutton RA. Rediscovery and redefinition of Malcolm Smith's Trimeresurus kanburiensis in Thailand, with a report of envenoming. Trans Royal Soc Trop Med Hygiene. 1992;86(1):95-9.

3. World Health Organization. Neglected tropical diseases. Geneva, Switzerland. Available at: https://www.who.int/neglected_diseases/en/.

Accessed on 25 February 2019.

4. Valladevi PS, Hossaini SB. Epidemiology, clinical profile of snake bite cases treated in Theni Medical College and Hospital. IOSR Journal of Dental and Medical Sciences. 2017;16: 48-50.

5. Hati AK, Mandal M, De MK, Mukherjee H, Hati RN. Epidemiology of snake bite in the district of Burdwan, West Bengal. J Ind Med Assoc. 1992;90(6):145-7.

6. Majumder D, Sinha A, Bhattacharya SK, Ram R, Dasgupta U, Ram A. Epidemiological profile of snake bite in South 24 Parganas district of West Bengal with 
focus on underreporting of snake bite deaths. Indian J Public Health. 2014;58:17-21.

7. Philip E. Snake bite and scorpion sting. In: Srivastava RN, eds. Jaypee Brothers; New Delhi: 1994; 28: $227-$ 234.

8. Shahmy S, Kularatne SA, Rathnayake SS, Dawson AH. A prospective cohort study of the effectiveness of the primary hospital management of all snakebites in Kurunegala district of Sri Lanka. PLoS Neglected Trop Dis. 2017;11(8):e0005847.

9. Majumder D, Sinha A, Bhattacharya S, Ram R, Dasgupta U, Ram A. Epidemiological profile of snake bite in south 24 Parganas district of West Bengal with focus on underreporting of snake bite deaths. Ind J Pub Heal. 2014;58(1):17-21.

10. Gupta YK, Peshin SS. Snake bite in India: current scenario of an old problem. J Clin Toxicol. 2014;4(1):182.

11. Pore SM, Kumbhar AV, Shah RD, Patil PT, Patankar SM, Herekar RN, et al. Use of anti-snake venom and clinical outcomes in snake envenomation: a prospective observational study. Int $\mathrm{J}$ Basic Clin Pharmacol. 2017;6:1472-6.

12. WHO Model Lists of Essential Medicines. Available at:

https://www.who.int/medicines/publications/essential medicines/en/ Accessed on 28th March 2019.

13. Ghosh S, Maisnam I, Murmu BK, Mitra PK, Roy A, Simpson ID. A locally developed snakebite management protocol significantly reduces overall anti snake venom utilization in West Bengal, India. Wilderness Env Med. 2008;19(4):267-74.

14. Monzavi SM, Afshari R, Salarian AA, Khoshdel AR. Effectiveness of a clinical protocol implemented to standardize the snakebite management in Iran: results of an interventional study. Available at: http://apamt.org/demo/wpcontent/uploads/2016/11/12th_Oral_Presentation_23. pdf. Accessed on 1 March 2019.

15. Borkar MS, Lahane CG, Kashid AA, Chavan SU, Uppod SG. Effect of low dose ASV with supportive care in poisonous snake bites in Marathwada region, Aurangabad, Maharashtra, India. Int J Sci Rep. 2015;1:307-12.

16. Warrell DA. Snake bite. Lancet. 2010;375(9708):7788.
17. Indian Herpetological Society. Available at: http://www.indianherps.org/. Accessed on 27 February 2019

18. Sarin K, Dutta TK, Vinod KV. Clinical profile and complications of neurotoxic snake bite and comparison of two regimens of polyvalent anti-snake venom in its treatment. Ind $\mathbf{J}$ Med Res. 2017;145(1):58.

19. Gaitonde BB, Bhattacharya S. An epidemiological survey of snake bite cases in India. Snake. 1980;12:129-33.

20. Standard Treatment Guidelines on Snakebite. Directorate General of Health Services, Ministry of Health and Family Welfare, Government of India With Technical Support from National health Systems Resource Centre; 2016. Available at: http://www.nhm.gov.in/nrhm-instate/520-standardtreatment-guidelines.html Accessed on 28th March 2019.

21. Murugan A, Ahmed S, Gani M. A retrospective study of snake bite envenomation in a tertiary care teaching hospital in Southern India. Int J Res Med Sci. 2015;3:2419-24.

22. Poudyal VP, Paudal KM, Rana NB, Adhikari S. A hospital-based study on snake bite poisoning in adults in the western region of Nepal. J Chitwan Med Coll. 2016;6(3):33-8.

23. Paul V, Pratibha S, Prahlad KA, Earali J, Francis S, Lewis F. High-dose anti-snake venom versus low-dose anti-snake venom in the treatment of poisonous snake bites-a critical study. J Assoc Physicians Ind. 2004;52:14-7.

24. Dan S, Mandal A, Das A, Chakrabarty S, Gangopadhyay T. A 6 months retrospective observational study to assess the rationality and effectiveness of snake bite management in a tertiary care teaching hospital of rural Bengal, India. Int $\mathbf{J}$ Basic Clin Pharmacol. 2018;7:2047-52.

Cite this article as: Mandal S, Mandal A, Chatterjee Y, Das S, Kundu S, Naser SM. A prospective analysis of adherence to standard treatment guidelines in management of snakebite in a tertiary care teaching hospital of rural Bengal, India. Int J Basic Clin Pharmacol 2019;8:849-55. 